\title{
A lightweight sensing platform for monitoring sleep quality and posture: a simulated validation study
}

\author{
Richard M. Kwasnicki ${ }^{1,2 *}$, George W. V. Cross ${ }^{1}$, Luke Geoghegan ${ }^{1}$, Zhiqiang Zhang ${ }^{2}$, Peter Reilly ${ }^{3}$, Ara Darzi², \\ Guang Zhong Yang ${ }^{2}$ and Roger Emery ${ }^{3}$
}

\begin{abstract}
Background: The prevalence of self-reported shoulder pain in the UK has been estimated at $16 \%$. This has been linked with significant sleep disturbance. It is possible that this relationship is bidirectional, with both symptoms capable of causing the other. Within the field of sleep monitoring, there is a requirement for a mobile and unobtrusive device capable of monitoring sleep posture and quality. This study investigates the feasibility of a wearable sleep system (WSS) in accurately detecting sleeping posture and physical activity.

Methods: Sixteen healthy subjects were recruited and fitted with three wearable inertial sensors on the trunk and forearms. Ten participants were entered into a 'Posture' protocol; assuming a series of common sleeping postures in a simulated bedroom. Five participants completed an 'Activity' protocol, in which a triphasic simulated sleep was performed including awake, sleep and REM phases. A combined sleep posture and activity protocol was then conducted as a 'Proof of Concept' model. Data were used to train a posture detection algorithm, and added to activity to predict sleep phase. Classification accuracy of the WSS was measured during the simulations.

Results: The WSS was found to have an overall accuracy of $99.5 \%$ in detection of four major postures, and $92.5 \%$ in the detection of eight minor postures. Prediction of sleep phase using activity measurements was accurate in $97.3 \%$ of the simulations. The ability of the system to accurately detect both posture and activity enabled the design of a conceptual layout for a user-friendly tablet application.

Conclusions: The study presents a pervasive wearable sensor platform, which can accurately detect both sleeping posture and activity in non-specialised environments. The extent and accuracy of sleep metrics available advances the current state-of-the-art technology. This has potential diagnostic implications in musculoskeletal pathology and with the addition of alerts may provide therapeutic value in a range of areas including the prevention of pressure sores.
\end{abstract}

Keywords: Shoulder, Pervasive, Monitoring, Posture, Sleep, Activity, Sensors, Wearables

\section{Background}

Pathologies affecting the shoulder are common within the population, often leading to a significant loss of function. UK prevalence of self-reported shoulder pain has been estimated at $16 \%$, with rates as high as $26 \%$ in the elderly [1]. Accounting for $2.36 \%$ of presentations to

\footnotetext{
*Correspondence: richard.kwasnicki07@imperial.ac.uk

${ }^{1}$ Department of Surgery and Cancer, Imperial College London, 10th Floor QEQM Building, St. Mary's Hospital, Praed Street, London W2 1NY, UK Full list of author information is available at the end of the article
}

general practitioners, it is the third most common musculoskeletal presenting complaint [2]. Shoulder pain has been found to lead to significant sleep disturbance, which consequentially has significant impact on quality of life. Further, sleeping posture has been implicated as a causative mechanism of certain shoulder pathologies, as well as having a detrimental impact on post-operative healing following musculoskeletal surgery.

Patients following shoulder surgery demonstrated greater pain intensity and duration in comparison to total hip and knee arthroplasty patient cohorts, with such pain 
having significantly greater interference with sleep and activities of daily living [3]. Smith et al. [4] demonstrated that $90 \%$ of patients were unable to sleep on their affected shoulder post-operatively, additionally $80 \%$ of patients with shoulder conditions without prior surgery were also unable to sleep on their affected shoulder [5].

It has been hypothesised that a lateral decubitus sleeping position can lead to increased shoulder pressure for extended periods of time, precipitating chronic pain [6]. Kempf et al. [7] demonstrated a significant correlation of $68 \%$ between the side of shoulder pain and preferred sleeping side, and hypothesised that manipulating sleeping posture could prevent further damage to the shoulder. Werner et al. [8] reported that the supine position resulted in significantly lower subacromial pressures compared to prone and lateral decubitus positions, demonstrating that sleep positions leading to active flexion, abduction and internal rotation should be avoided during recovery.

Monitoring sleep quality may provide broad insight into general health status of patients [9-13], with extension of monitoring into patients with shoulder pathologies seeming logical given the high prevalence of sleep disturbance, anxiety and depression [14]. In the setting of shoulder pathology, sleep activity and posture provide surrogate markers for sleep quality and limb positioning. The current gold standard for sleep monitoring is polysomnography (PSG); however, there have been technological advancements to produce less intrusive methods of monitoring sleep, which can be used in a more natural sleep setting. These include actigraphy (physical activity), heart rate variability (HRV), and smartphone applications. Actigraphy and HRV are currently the only methods that have been validated to measure sleep quality to a high degree of accuracy, ranging from 82-97 and $57-93 \%$, respectively [15-21].

Alongside sleep quality, posture monitoring during sleep provides clinically valuable information regarding the arrangement of limbs and resultant joint angles and likely pressures. Electrocardiogram (ECG) waveforms, pressure sensors and ultra-wide band (UWB) frequency technology have all previously been used in the determination of sleep posture, though their efficacy in the patients with shoulder pain is yet to be established [2227]. Joint angle measurements provide another dimension to sleep posture monitoring and have demonstrated efficacy in the orthopaedic patient cohort. The current main methodologies in the measurement of joint angles include inertial sensors, universal goniometer and smartphone applications, with inertial sensors attracting the greatest interest within the literature [28-34]. However, traditional emphasis has been placed upon the refinement of wearable sensors in isolation. In contrast, the current work aimed to develop a multi-sensor system architecture combining wearable sensors, communication technology and data analytics in a single platform capable of ambulatory monitoring. Herein, the presented study aims to investigate the accuracy of a wearable sleep system (WSS) in the detection of sleep quality and posture.

\section{Methods}

\section{Study overview}

A series of laboratory-based simulations were designed to assess the ability of WSS to detect sleeping posture and activity. Healthy adult subjects were recruited locally at the Hamlyn Centre (Imperial College London) and excluded if they demonstrated active shoulder pathology or reported upper body mobility limitation of any sort. Ethical approval was gained from the NRES Committee London-Dulwich on 19th November 2013 (REC Reference: 10/H0808/124). Informed consent was obtained directly from all study participants.

Sleeping posture was investigated by asking healthy individuals to assume a variety of predefined sleeping positions. Sleep activity was investigated by asking subjects to simulate a typical sleeping period progressing through stages of sleep and replicating associated activity at each stage.

\section{Wearable sleep system (WSS)}

A bespoke WSS was designed consisting of a small wearable sensor positioned on each arm and the chest, (seen in Fig. 1) communicating wirelessly to a local processing unit (in this study was a laptop computer) using a radiofrequency transceiver. The node platform consists of a TI MSP430 ultra low power processor, a Chipcon CC2420 RF module for wireless communications and a light weight Li-ion polymer battery. The node is integrated with an Analog Devices ADXL330 for measurement of 3D acceleration, an InvenSense ITG-3200 digital gyroscope for 3D angular velocity measurement and a Honeywell HMC5843 for 3D magnetic field measurement as previously described. The whole sensor node measures $20 \times 30 \times 17 \mathrm{~mm}$ with a weight of $10 \mathrm{~g}$ and has previously been shown effective in the quantitative analysis of human body movements [35].

\section{Posture monitoring}

Participants were first asked to lie on the bed and assume eight postures in a known order, demonstrated in Fig. 2, to allow sensor calibration. Each posture was maintained for $10 \mathrm{~s}$ before participants were instructed to assume the next predefined posture. To facilitate an assessment of blinded classification accuracy, each participant re-enacted the postures in a randomised order. The 


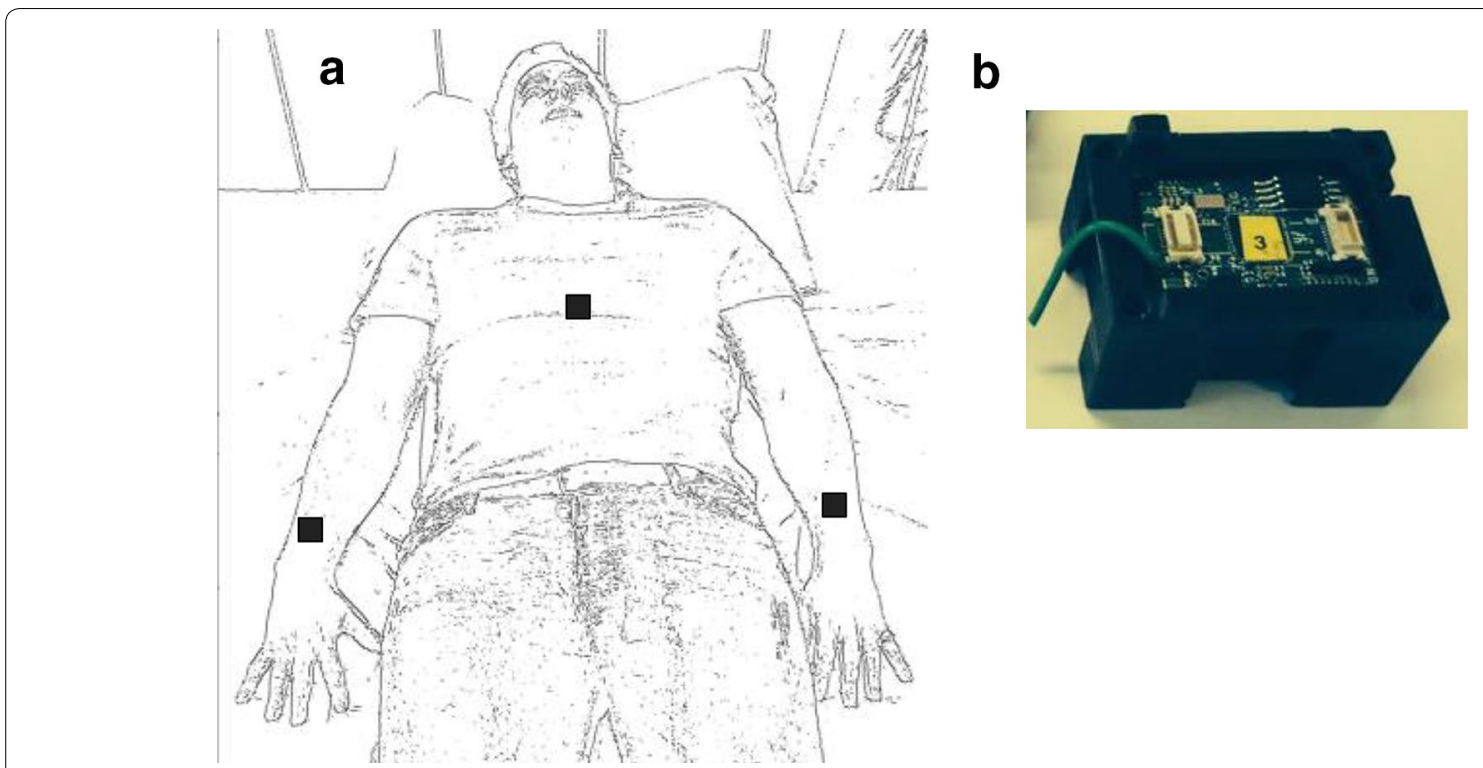

Fig. 1 The WSS sensor platform. a Schematic representation of WSS sensors placement, with one on each arm and one on the trunk. $\mathbf{b}$ Structural representation of the WSS sensors used

randomised sequence consisted of 16 total postures with each original posture performed twice by participants. A broad range of postures were chosen to determine the ability of the WSS to recognise both major and minor movements that may be employed by patients in the clinical setting. In order to direct the selection of such positions, focus groups were held with study participants to identify common sleeping positions amongst individuals without shoulder pathology. Further sleeping positions in those with shoulder pain were identified by clinical authors through consultation with patients suffering with shoulder pathologies.

\section{Sleep quality monitoring}

Participants were asked to perform a semi-structured simulation of the three sleep stages (awake, sleep and REM sleep) based upon the degree of major and minor transition movement permitted as outlined in the sleep stage simulation protocol, Table 1. Major transitions were defined as movement between the four main postures: right slide, left side, supine and prone. Minor transitions were defined as isolated movement of the limbs within each of the four main postures. Major and minor transition movements were standardised before recording commenced through verbal explanation and the use of a visual aid, Fig. 2. Each sleep stage was simulated for 2 min with a sequential order of A, B, C, $\mathrm{B}, \mathrm{C}, \mathrm{B}, \mathrm{A}$, providing $14 \mathrm{~min}$ of recording activity for each participant, where A represents awake, B represents sleep, and $C$ represents REM sleep. Our string of movements therefore simulates progression throughout natural sleep. Table 1 outlines the degree of movement permitted in each of the sleep stages A, B and C. The sleep stage simulation protocol was developed in view of the limited battery life of the WSS (14 min) and was designed to broadly simulate differing stages of sleep across the constrained time period.

\section{Combined proof of concept model}

A single participant completed a simulation including combination of both posture and activity changes to assess the validity of WSS as a future platform for total sleep monitoring. Sensors were placed as described in Fig. 1 and the participant was asked to calibrate the sensors with known body position as previously described. Immediately after completion of all eight postures the subject was then asked to perform the same three simulated sleep phases.

\section{Data analysis}

Data from tri-axial accelerometer, gyroscope, and magnetometer for all three sensors were integrated and wirelessly sent to a receiving laptop. An algorithm was developed to allow the training of a computerised posture classifier based on calibration data (see Additional file 1). Data were analysed on MATLAB ${ }^{\circledR}$ (MathWorks Version R2014a (8.3.0.532) to quantify both individual and overall accuracy and error. For activity, a surrogate for sleep phase, accelerometer values from each of 


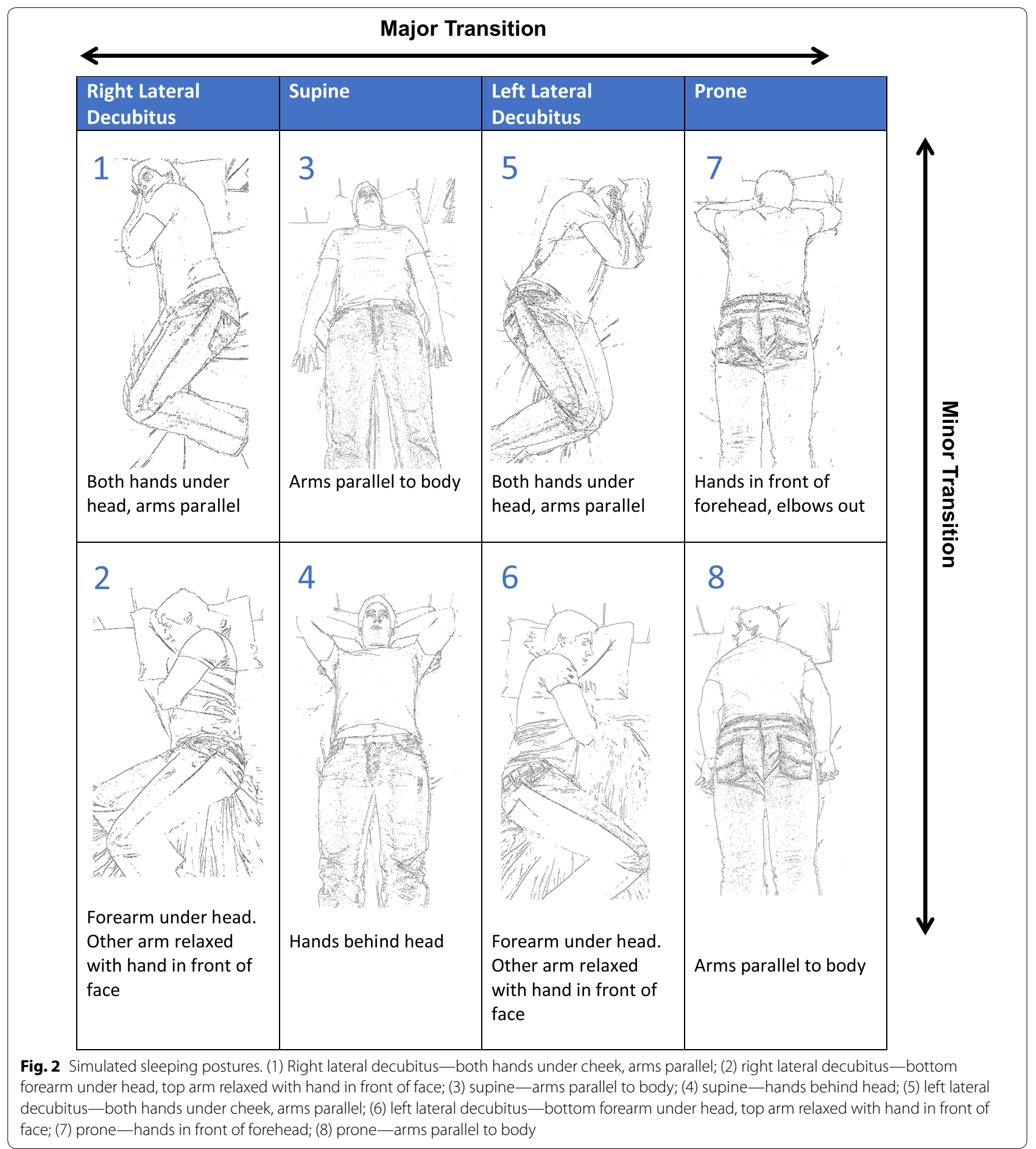

the three sensors were combined to estimate activity level using data variance, i.e. the magnitude of 3-dimensional movement. Subject-specific activity thresholds were derived from study data and used to determine the simulated sleep phase. The nested IF function:=IF
$(F 3>0.14$, "3”, IF $(F 3>0.058$, ,", IF $(F 3<0.058$, “1”)) was used to quantify activity levels and corresponding sleep stages. To calculate the percentage of time, the participant spent in each phase the COUNTIF function $=$ COUNTIF $(\mathrm{H} 3: \mathrm{H} 24509,3)$ was run across all data. 
Table 1 Sleep stage simulation protocol

\begin{tabular}{llll}
\hline $\begin{array}{l}\text { Simulated } \\
\text { sleep stage }\end{array}$ & $\begin{array}{l}\text { Major } \\
\text { transition } \\
\text { permitted }\end{array}$ & $\begin{array}{l}\text { Minor } \\
\text { transition } \\
\text { permitted }\end{array}$ & $\begin{array}{l}\text { Movement } \\
\text { between transitions } \\
\text { permitted }\end{array}$ \\
\hline Awake & Every $30 \mathrm{~s}$ & No & Yes \\
Sleep & No & Every minute & Limited \\
REM sleep & No & No & No
\end{tabular}

The degree of movement permitted during the each of the simulated sleep stages is outlined along with corresponding major and minor transition frequencies where appropriate

\section{Statistical analysis}

Statistical tests were used to determine if any of the participants or postures demonstrated particularly high and low accuracy levels. The non-parametric test KruskalWallis one-way non-parametric analysis of variance (KW-ANOVA) was used to initially detect any outliers. Outliers were further compared against group averages using the Mann-Whitney $U$ Test. Analyses were performed using SPSS version 20.0 for Windows. The statistical significance level was set at $P<0.05$.

\section{Results}

Sixteen healthy subjects were recruited, ten participating in the posture protocol, five in the activity protocol, and one for the proof on concept simulation. There were seven females and nine males with a mean age of 25 years old. No major technical issues arose and datasets were available for all participants recruited.

\section{Posture}

Across 10 participants, the WSS platform showed an overall classification accuracy of $99.5 \%$ for detecting the four main sleeping postures: right, supine, left, and prone. Classification accuracy across all eight postures was $92.5 \%$ (Fig. 3).

The distinction between postures 1 and 2 was the most difficult to classify, followed by postures 5 and 6 . After KW-ANOVA, the mean rank suggested postures 1, 2 and 6 to be outliers. A Mann-Whitney $U$ test found no statistical significance between the classification accuracy of these postures compared to the others. Between participants, the classification accuracy varied from 84.3 to $100 \%$.

\section{Activity}

Activity classification across five participants estimated $28.5 \%$ of the time spent awake (28.6\% simulated), $42.6 \%$ of the time spent asleep ( $42.9 \%$ simulated) and $26.6 \%$ of the time spent in REM (28.6\% simulated). The activity measured for the simulated sleep of one participant are shown in Fig. 4, represented as the combined coefficient

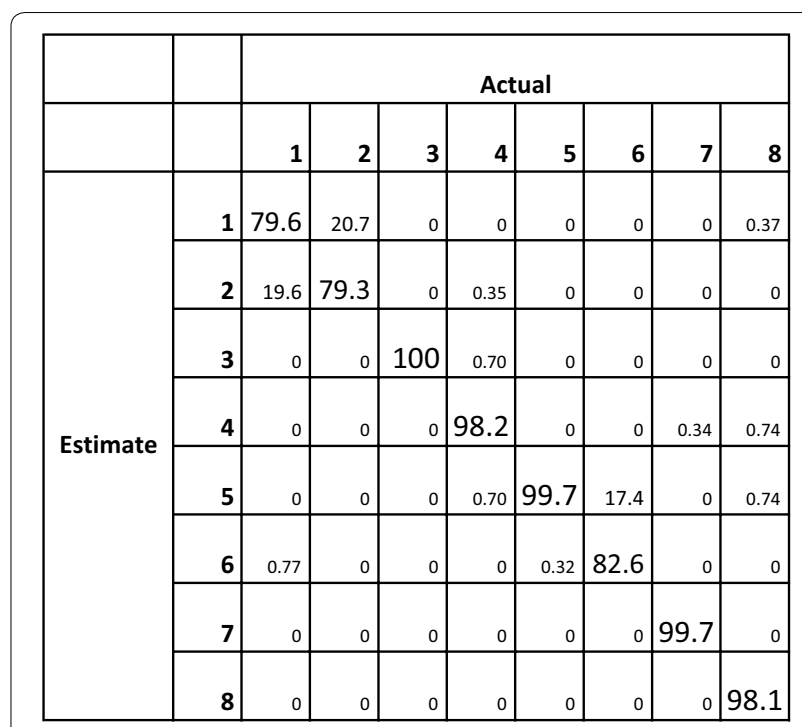

Fig. 3 Classification matrix representing the percentage accuracy for the eight main postures

of variation calculated from each of the 3 accelerometer axes of each sensor.

\section{Discussion}

This study demonstrates the high accuracy levels achievable for monitoring physical activity and postures during sleep with a wearable sensor platform. The WSS was found to have an overall accuracy of $99.5 \%$ in the detection of four main postures, which was mostly maintained when detecting eight postures producing an accuracy of 92.5\%. Accuracy was acceptable across all subjects with the least accurate being $84.3 \%$. The platform could predict simulated sleep phases (awake, sleep, REM) using arm and trunk activity measurements. The ability of the system to detect both posture and activity was exhibited in a proof of concept dataset, along with a conceptual layout for a tablet application to be used by both doctors and patients (Fig. 5).

The 4-posture classification accuracy (99.5\%) compares well to other papers in the field. Hsia et al. [23] used a Bayesian Classification with pressure sensors finding an overall accuracy of $81.4 \%$. The use of a wireless identification and sensing platform by Hoque et al. [36] gained a $94.4 \%$ accuracy in detection of the four postures, whilst $\mathrm{Ni}$ et al. [27] received similar results to this study in their use of UWB tags combined with a pressure sensor matrix, at approximately 99\% accuracy. For eight postures, the WSS demonstrated an accuracy of $92.5 \%$. Two studies using embedded pressure sensors, one considering five postures, and the other nine, gained accuracies of 97.7 and $94 \%$, respectively [24, 25]. Although both these 


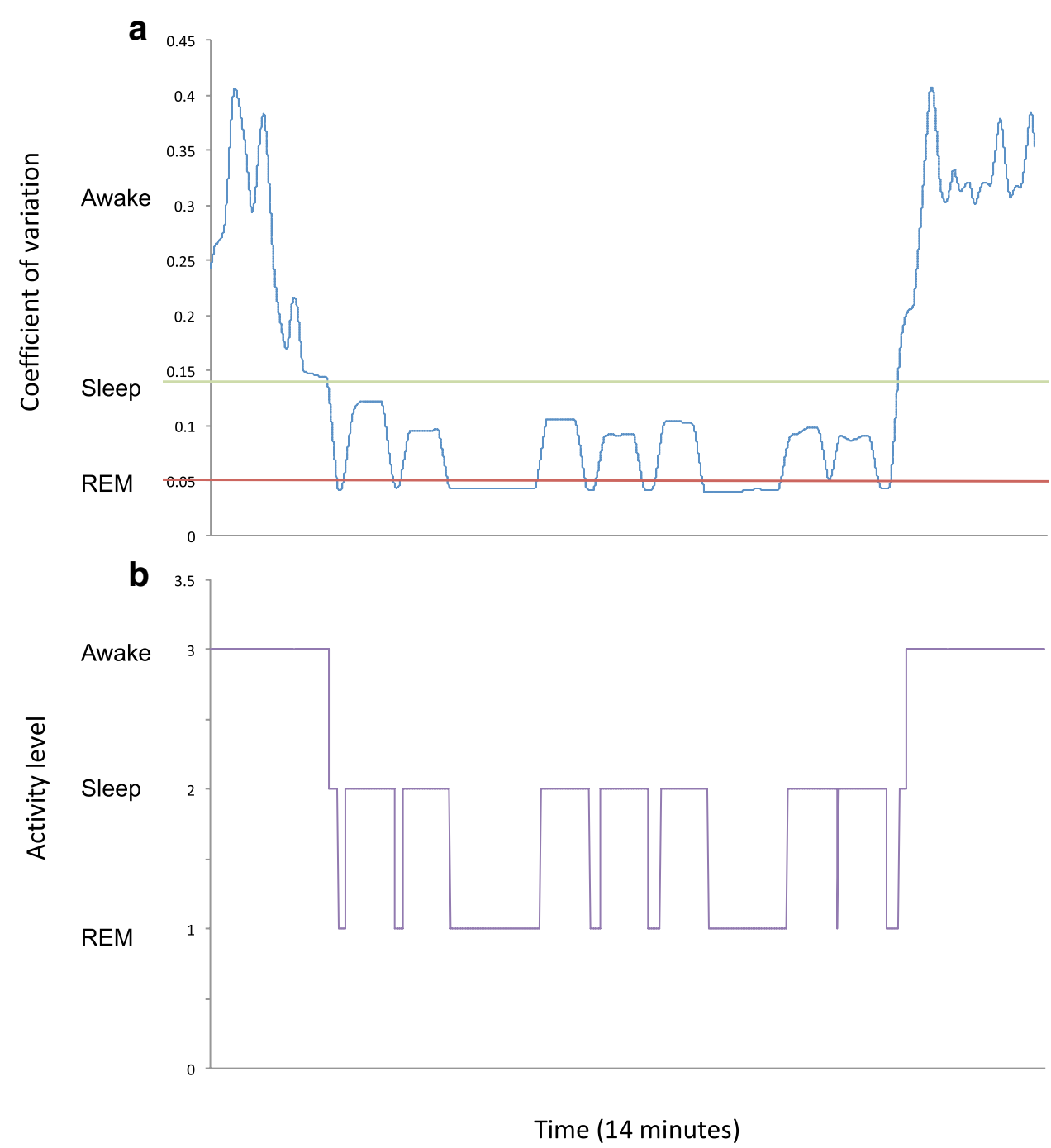

Fig. 4 Graphical representation of the various stages of sleep quantified using the WSS. a Represents the combined coefficient of variation from all three sensors; $\mathbf{b}$ represents the separation of (a) into the three phases: 1 (REM), 2 (sleep) and 3 (awake)

papers gained a higher classification accuracy compared to our $92.5 \%$, pressure systems were embedded within mattresses, with the system described remaining the most accurate and advanced wearable platform.

Distinction between two right-sided postures (1 and 2) yielded the greatest identification error during our study. These postures are very similar, with the only difference being $90^{\circ}$ rotation at the shoulder in one arm. Therefore, it is possible that the error in these postures is due to poor user compliance with the participant not recreating the posture performed during calibration. These findings are replicated on the left side. The possibility of poor participant compliance is further highlighted by the fact that in 2 out of 10 participants the sensors were $100 \%$ accurate, whilst other participants had accuracy levels as low as $84.3 \%$. That said, in a true clinical model similar issues are likely to occur, but whether minor differences in position will affect the utility of the information in currently unclear.

The WSS could measure the duration of each sleep phase with high accuracy, only the REM phase having a greater than $1 \%$ deviation from the predicted, leading to an overall accuracy of $97.3 \%$. This validates the ability of WSS to assess activity levels of a person whilst they are sleeping, facilitating the assessment of sleep quality in a natural environment. This compares well to the actigraph sensor, with the added benefit of simultaneous posture detection. Chang et al. [37] are the only other group who have been able to provide a platform that is able to detect both sleeping posture and activity levels. A tri-axial accelerometer was used on the chest for posture detection, combined with ECG recording for sleep stage 


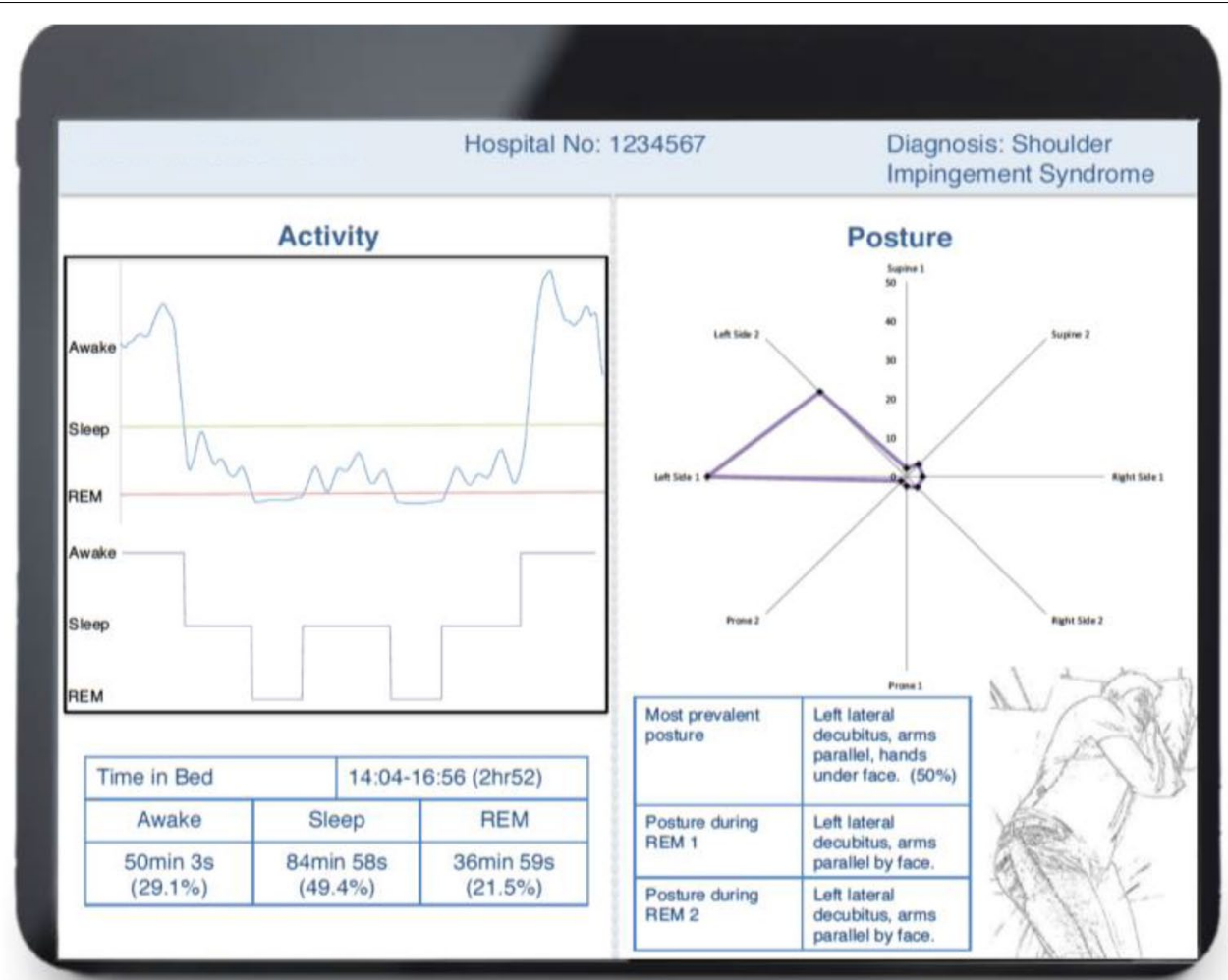

Fig. 5 Proof of concept output from the pervasive sleep sensor platform presented as part of a conceptual application interface. Reported data include demographics, activity levels with corresponding time intervals and relative posture for utilisation by clinicians and patients

monitoring [37]. The method described in this paper provides additional information regarding the position of the upper limbs which is of particular interest in musculoskeletal pathology.

The envisaged clinical impact of the WSS is primarily diagnostic, but with small adjuncts could become therapeutic. Widening access of sleep monitoring beyond specialist facilities would allow patients suffering from upper limb symptoms to consider if sleep position may be a contributing factor. It also facilitates research into the sleep behaviour of post-operative patients, which may give insight into why some experience delay in recovery. The addition of an alert function based on pre-set criteria such as sleeping in one position for too long, might allow for therapeutic utility, e.g. following shoulder arthroplasty. In the broader healthcare setting use of such systems might help prevent pressure sores by alerting carers when patients have been in one position for a certain length of time.

The interpretation and application of the results should be done so in the context of the study limitations. Despite the promising accuracy demonstrated by the WSS, our data were only collected from a cohort of healthy patients measured over $14 \mathrm{~min}$ of simulated sleep. Future comparative studies seeking to replicate such findings in a cohort of patients with shoulder pathology over a natural sleep cycle would prove useful in determining if similar results can be obtained in those with shoulder pain and concurrent sleep disturbance. Of note, the sleep postures chosen were designed to capture potential movements replicated in a clinical cohort. Future comparative studies will yield valuable data regarding actual preferred sleep positions in patients with shoulder pathology. As a validation study, the current work proves useful in providing preliminary data to inform the design of future comparative studies.

The main technical limitations of the WSS include battery life and sensor size. Unfortunately, the current WSS battery only lasts for $30 \mathrm{~min}$, making overnight use currently unfeasible. To mitigate this, protocols were tailored accordingly, allowing the simulation of sequences to represent part of a night's sleep. As prototypes, the sensors are cumbersome $(20 \times 30 \times 17 \mathrm{~mm})$ leading to potential discomfort. Both limitations could be overcome with formal sensor design and packaging, and optimising sensor settings with regard to frequency of data capture and transmission. The study participant demographic was not in keeping with that of the clinical cohort; however, this is unlikely to affect the potential utility. 


\section{Conclusions}

This work demonstrates the accuracy of a wireless sensor platform to detect sleeping posture and quality. This has potential for use in patients with musculoskeletal pathology, as well as other healthcare applications such as pressure sore prevention. Ultimately, it is hoped that such sensor platforms could provide a low cost, mobile sleep laboratory, which could facilitate a greater number of sleep studies offering insight into disease processes, and help tailor the holistic management of patients with musculoskeletal disease.

\section{Additional file}

Additional file 1. Posture classification algorithm. (A) The main part of the algorithm that runs in MATLAB ${ }^{\circledR}$ used to determine posture classification. (B) A brief mechanistic overview of the algorithm.

\section{Abbreviations}

WSS: wearable sleep system; REM: rapid eye movements; PSG: polysomnography; UWB: ultra-wide band; HRV: heart rate variability; ECG: echocardiogram; KW-ANOVA: Kruskal-Wallis one-way non-parametric analysis of variance.

\section{Authors' contributions}

RK and GC designed the project, collected, and analysed data. RK, LG, and GC developed the manuscript. ZZ and GZY assisted with software development and provided technical support throughout the duration of the project. PR, $A D$, and RE provided senior clinical input for the design and conduct of the project. All authors reviewed the manuscript. All authors read and approved the final manuscript.

\section{Author details \\ ${ }^{1}$ Department of Surgery and Cancer, Imperial College London, 10th Floor QEQM Building, St. Mary's Hospital, Praed Street, London W2 1NY, UK. ${ }^{2}$ The Hamlyn Centre, Institute of Global Health Innovation, Imperial College Lon- don, London, UK. ${ }^{3}$ Imperial College Healthcare NHS Trust, London, UK.}

\section{Acknowledgements}

The authors would like to thank all volunteers for their time and involvement with the study.

\section{Competing interests}

The authors certify that they have no affiliations nor financial involvement with any organisation or entity with direct financial interests in the subject matter of this article.

\section{Availability of data and materials}

The datasets used and analysed during the current study are available from the corresponding author on reasonable request.

\section{Consent for publication}

Not applicable.

\section{Ethics approval and consent to participate}

Ethical approval was gained from NRES Committee London-Dulwich, on 19th November 2013, REC Reference: 10/H0808/124.

\section{Funding}

Supported by (1) Engineering and Physical Sciences Research Council (EPSRC)_ESPRIT project; (2) National Institute for Health Research (NIHR) Biomedical Research Centre based at Imperial College Healthcare NHS Trust and Imperial College London.

\section{Publisher's Note}

Springer Nature remains neutral with regard to jurisdictional claims in published maps and institutional affiliations.

Received: 17 March 2018 Accepted: 18 May 2018

Published online: 30 May 2018

\section{References}

1. Urwin M, Symmons D, Allison T, Brammah T, Busby H, Roxby M, Simmons A, Williams G. Estimating the burden of musculoskeletal disorders in the community: the comparative prevalence of symptoms at different anatomical sites, and the relation to social deprivation. Ann Rheum Dis. 1998:57(11):649-55.

2. Linsell L, Dawson J, Zondervan K, Rose P, Randall T, Fitzpatrick R, Carr A. Prevalence and incidence of adults consulting for shoulder conditions in UK primary care; patterns of diagnosis and referral. Rheumatology (Oxford). 2006:45(2):215-21.

3. Lindberg MF, Grov EK, Gay CL, Rustoen T, Granheim TI, Amlie E, Lerdal A. Pain characteristics and self-rated health after elective orthopaedic surgery-a cross-sectional survey. J Clin Nurs. 2013;22(9-10):1242-53.

4. Smith KL, Harryman DT 2nd, Antoniou J, Campbell B, Sidles JA, Matsen FA 3rd. A prospective, multipractice study of shoulder function and health status in patients with documented rotator cuff tears. J Shoulder Elbow Surg. 2000;9(5):395-402.

5. Largacha M, Parsons IT, Campbell B, Titelman RM, Smith KL, Matsen F 3rd. Deficits in shoulder function and general health associated with sixteen common shoulder diagnoses: a study of 2674 patients. J Shoulder Elbow Surg. 2006;15(1):30-9.

6. Zenian J. Sleep position and shoulder pain. Med Hypotheses. 2010;74(4):639-43.

7. Kempf B, Kongsted A. Association between the side of unilateral shoulde pain and preferred sleeping position: a cross-sectional study of 83 Danish patients. J Manipulative Physiol Ther. 2012;35(5):407-12.

8. Werner CM, Ossendorf C, Meyer DC, Blumenthal S, Gerber C. Subacromial pressures vary with simulated sleep positions. J Shoulder Elbow Surg. 2010;19(7):989-93.

9. Baldwin CM, Griffith KA, Nieto FJ, O'Connor GT, Walsleben JA, Redline S. The association of sleep-disordered breathing and sleep symptoms with quality of life in the Sleep Heart Health Study. Sleep. 2001;24(1):96-105.

10. Becker HF, Jerrentrup A, Ploch T, Grote L, Penzel T, Sullivan CE, Peter $J \mathrm{H}$. Effect of nasal continuous positive airway pressure treatment on blood pressure in patients with obstructive sleep apnea. Circulation. 2003;107(1):68-73.

11. Khan A, Latif F, Hawkins B, Tawk M, Sivaram CA, Kinasewitz G. Effects of obstructive sleep apnea treatment on left atrial volume and left atrial volume index. Sleep Breath. 2008;12(2):141-7.

12. Punjabi NM, Shahar E, Redline S, Gottlieb DJ, Givelber R, Resnick HE. Sleep-disordered breathing, glucose intolerance, and insulin resistance: the Sleep Heart Health Study. Am J Epidemiol. 2004;160(6):521-30.

13. Shahar E, Whitney CW, Redline S, Lee ET, Newman AB, Nieto FJ, O'Connor GT, Boland LL, Schwartz JE, Samet JM. Sleep-disordered breathing and cardiovascular disease: cross-sectional results of the Sleep Heart Health Study. Am J Respir Crit Care Med. 2001;163(1):19-25.

14. Cho CH, Jung SW, Park JY, Song KS, Yu KI. Is shoulder pain for three months or longer correlated with depression, anxiety, and sleep disturbance? J Shoulder Elbow Surg. 2013;22(2):222-8.

15. Belanger ME, Bernier A, Paquet J, Simard V, Carrier J. Validating actigraphy as a measure of sleep for preschool children. J Clin Sleep Med. 2013:9(7):701-6.

16. Paquet J, Kawinska A, Carrier J. Wake detection capacity of actigraphy during sleep. Sleep. 2007:30(10):1362-9.

17. Meltzer LJ, Walsh CM, Traylor J, Westin AM. Direct comparison of two new actigraphs and polysomnography in children and adolescents. Sleep. 2012;35(1):159-66.

18. Dick R, Penzel T, Fietze I, Partinen M, Hein H, Schulz J. AASM standards of practice compliant validation of actigraphic sleep analysis from SOMNOwatch versus polysomnographic sleep diagnostics shows high 
conformity also among subjects with sleep disordered breathing. Physiol Meas. 2010;31(12):1623-33.

19. Pollak CP, Tryon WW, Nagaraja H, Dzwonczyk R. How accurately does wrist actigraphy identify the states of sleep and wakefulness? Sleep. 2001;24(8):957-65.

20. Lewicke AT, Sazonov ES, Schuckers SA. Sleep-wake identification in infants: heart rate variability compared to actigraphy. In: Conference proceedings: annual international conference of the IEEE engineering in medicine and biology society IEEE engineering in medicine and biology society annual conference. 2004. vol. 1, p. 442-5.

21. Ebrahimi F, Setarehdan SK, Ayala-Moyeda J, Nazeran H. Automatic sleep staging using empirical mode decomposition, discrete wavelet transform, time-domain, and nonlinear dynamics features of heart rate variability signals. Comput Methods Programs Biomed. 2013;1 12(1):47-57.

22. Hsia C-C, Hung Y-W, Chiu Y-H, Kang C-H. Bayesian classification for bed posture detection based on kurtosis and skewness estimation. In: e-health Networking, applications and services, 2008 HealthCom 2008 10th international conference on: 2008: IEEE. 2008. p. 165-8.

23. Hsia C, Liou K, Aung A, Foo V, Huang W, Biswas J. Analysis and comparison of sleeping posture classification methods using pressure sensitive bed system. In: Engineering in medicine and biology society, 2009 EMBC 2009 annual international conference of the IEEE: 2009, IEEE. 2009. p. 6131-4.

24. Huang W, Wai AAP, Foo SF, Biswas J, Hsia C-C, Liou K. Multimodal sleeping posture classification. In: Pattern recognition (ICPR), 2010 20th international conference on 2010: IEEE. 2010. p. 4336-9.

25. Yousefi R, Ostadabbas S, Faezipour M, Farshbaf M, Nourani M, Tamil L, Pompeo M. Bed posture classification for pressure ulcer prevention. In: Engineering in medicine and biology society, EMBC, 2011 annual international conference of the IEEE: 2011, IEEE. 2011. p. 7175-8.

26. Schrempf A, Schoßleitner G, Blaha A, Leipold S: Measuring nightly activity, body weight and body weight change rate with a sensor equipped bed. In: Engineering in medicine and biology society (EMBC), 2010 annual international conference of the IEEE: 2010, IEEE. 2010. p. 2151-4.

27. Ni H, Abdulrazak B, Zhang D, Wu S, Zhou X, Miao K, Han D. Multi-modal non-intrusive sleep pattern recognition in elder assistive environment.
In: Donnelly M, Paggetti C, Nugent C, Mokhtari M. Impact analysis of solutions for chronic disease prevention and management: 10th international conference on smart homes and Health Telematics, ICOST 2012, Artiminio, Italy, June 12-15, 2012 Proceedings. Berlin: Springer Berlin Heidelberg; 2012. p. 132-9.

28. Gibbs PT, Asada H. Wearable conductive fiber sensors for multi-axis human joint angle measurements. J Neuroeng Rehabil. 2005;2(1):7.

29. Dejnabadi H, Jolles BM, Aminian K. A new approach to accurate measurement of uniaxial joint angles based on a combination of accelerometers and gyroscopes. IEEE Trans Biomed Eng. 2005;52(8):1478-84.

30. Piotte F, Gravel D, Nadeau S, Moffet H, Bédard C. Reliability of arthrometric measurement of shoulder lateral rotation movement in healthy subjects. Physiother Theory Pract. 2007;23(3):169-78.

31. Favre J, Jolles B, Aissaoui R, Aminian K. Ambulatory measurement of 3D knee joint angle. J Biomech. 2008:41 (5):1029-35.

32. Piriyaprasarth $P$, Morris ME, Winter A, Bialocerkowski AE. The reliability of knee joint position testing using electrogoniometry. BMC Musculoskelet Disord. 2008;9(1):6

33. Cooper G, Sheret I, McMillian L, Siliverdis K, Sha N, Hodgins D, Kenney L, Howard D. Inertial sensor-based knee flexion/extension angle estimation. J Biomech. 2009:42(16):2678-85.

34. Jenny JY. Measurement of the knee flexion angle with a Smartphoneapplication is precise and accurate. J Arthroplasty. 2013;28(5):784-7.

35. Zhang Z-Q, Pansiot J, Lo B, Yang G-Z. Human back movement analysis using bsn. In: Body sensor networks (BSN), 2011 international conference on: 2011, IEEE. 2011. p. 13-8.

36. Hoque E, Dickerson RF, Stankovic JA. Monitoring body positions and movements during sleep using wisps. In: Wireless Health 2010: ACM; 2010. p. 44-53.

37. Chang K-M, Liu S-H. Wireless portable electrocardiogram and a tri-axis accelerometer implementation and application on sleep activity monitoring. Telemed E Health. 2011;17(3):177-84.
Ready to submit your research? Choose BMC and benefit from:

- fast, convenient online submission

- thorough peer review by experienced researchers in your field

- rapid publication on acceptance

- support for research data, including large and complex data types

- gold Open Access which fosters wider collaboration and increased citations

- maximum visibility for your research: over $100 \mathrm{M}$ website views per year

At $\mathrm{BMC}$, research is always in progress.

Learn more biomedcentral.com/submissions 